Théologiques

Théologiques

\title{
La question de Dieu dans la sociologie
}

\section{Nicole Laurin}

Volume 6, numéro 2, octobre 1998

Dieu interdit

URI : https://id.erudit.org/iderudit/024960ar

DOI : https://doi.org/10.7202/024960ar

Aller au sommaire du numéro

\section{Éditeur(s)}

Faculté de théologie de l'Université de Montréal

\section{ISSN}

1188-7109 (imprimé)

1492-1413 (numérique)

Découvrir la revue

\section{Citer cet article}

Laurin, N. (1998). La question de Dieu dans la sociologie. Théologiques, 6(2), 25-32. https://doi.org/10.7202/024960ar

\section{Résumé de l'article}

Dieu est désormais absent de la sociologie. Il a quitté d'abord la théorie sociologique générale et par la suite, il s'est retiré de la sociologie religieuse. De surcroît, en tant qu'élément de l'idéologie et source de mobilisation

intellectuelle et politique, il est tombé en désuétude. Au moment où la théologie chrétienne cherche son inspiration du côté des sciences et, particulièrement, des sciences humaines, la sociologie n'a donc plus rien à lui offrir. À part le fait social, dépouillé de ses oripeaux, ramené à sa misère et son absurdité. Le fait social dans sa pesanteur. d'utilisation que vous pouvez consulter en ligne.

https://apropos.erudit.org/fr/usagers/politique-dutilisation/ 
Théologiques 6/2 (1998) 25-32.

\title{
La question de Dieu dans la sociologie
}

\author{
Nicole LAURIN \\ Département de sociologie \\ Université de Montréal
}

La sociologie est athée. Elle se construit sans Dieu parce qu'elle n'a nul besoin de lui comme hypothèse fondatrice ou comme garant de la connaissance. Néanmoins, Dieu se présente-t-il sur son chemin, elle n'hésite pas à se l'approprier. Dieu devient alors une réalité sociale : il est soumis à une méthode et livré à un regard qui le produit comme objet dans le champ propre de la discipline. Toute autre issue pour Dieu, dans la sociologie, relève du "péché contre l'esprit ", en l'occurrence contre les règles et les usages de la pensée scientifique. La sociologie, il va sans dire, ne prétend pas au statut de science exacte ou expérimentale au même titre que la physique, par exemple, mais elle n'aime pas pour autant qu'on la confonde avec la littérature, la philosophie, la politique ou d'autres modes de connaissance. L'objet de la sociologie doit être conquis, souvent de haute lutte, sur le sens commun, la multiplicité des évidences et des apparences qui recouvrent cet objet. Sa démarche ne s'arrête pas là, il faut encore reconstruire l'objet selon les concepts et les théories de la discipline, pour permettre l'analyse et l'explication ${ }^{1}$. Ainsi de Dieu.

La question de Dieu dans la sociologie est très éloignée de mes préoccupations ordinaires. Aussi, ai-je tout d'abord refusé de m'aventurer sur ce terrain où je n'ai nulle compétence. L'insistance de plusieurs membres du comité de rédaction de Théologiques a eu raison de mes résistances. Toutefois, c'est au cours d'une réunion amicale chez Stephen Schecter, a l'occasion de la fête du Hanukah, que mon texte a pris forme virtuellement grace aux questions et aux commentaires passionnés de Anne-Marie Pelletier, Rebecca Augenfeld, Jean-Pierre Bourdouxe et Pierre Feuvrier. Je garde un souvenir ému de cet échange.

1 Les questions du statut scientifique de la sociologie, de la nature de son objet et de son mode de connaissance sont étudiées par plusieurs 
Aux origines de la sociologie, il occupe une place centrale dans la théorie. Historiquement, l'œuvre des fondateurs de la discipline participe à l'élaboration, hors du discours religieux et à contre-courant de ce discours, d'une réflexion sur la société inaugurée précédemment dans la philosophie politique. Sans doute aussi la société est-elle perçue par les premiers sociologues comme une énigme, un mystère à déchiffrer; sous ce rapport, elle demeure associée à Dieu. Selon la "loi des trois états" de l'humanité, proposée au XIX ${ }^{\mathrm{e}}$ siècle par Auguste Comte, la forme originelle de la société est théologique parce que Dieu y représente le principe premier d'explication ${ }^{2}$. Par conséquent les prêtres y détiennent le pouvoir spirituel. Cette société symbolise l'enfance de l'humanité et elle sert, dans la théorie, à faire valoir la pensée positiviste, associée à l'industrie moderne. D'ailleurs, à ce stade, la sociologie devient, selon Comte, la base du pouvoir spirituel et l'humanité remplace Dieu comme objet de la religion. Karl Marx, de son côté, s'efforce d'analyser sociologiquement la question de Dieu dans la foulée d'une critique qui dépasse, de loin, la thèse de "l'opium du peuple " à laquelle on a réduit sa pensée sur la religion ${ }^{3}$. Marx y voit une "nébuleuse ", un monde céleste de beauté, de justice et de bienveillance, dont il faut cependant extraire le "noyau terrestre». Non qu'il prétende, comme Feuerbach, restituer à "l'homme " son essence tout entière absorbée par le concept de Dieu ${ }^{4}$ mais parce qu'il veut comprendre comment et pourquoi ce monde se détache de la terre et se retire si

auteurs. On consultera, parmi les ouvrages les plus récents, celui de Jacques HAMEL, Précis d'épistémologie de la sociologie. Paris, L'Harmattan, 1997.

2 Auguste COMTE, Cours de philosophie positive, publié de 1830 à 1842 , 60 leçons en 6 volumes. Voir la synthèse de Jean LACROIX, La sociologie d'Auguste Comte. Paris, Presses universitaires de France, 1 ère édition en 1956.

3 Voir, en particulier, de Karl MARX, La question juive, 1844; Pour une critique de la philosophie du droit de Hegel, 1844; La Sainte famille, 1845; L'Idéologie allemande, 1845; dans Euvres, III (Philosophie). Paris, Gallimard, Bibliothèque de la Pléiade, 1982.

4 Ludwig FEUERBACH, L'Essence du christianisme. Paris, Maspero, 1968, (première édition en langue allemande, 1841). 
loin et si haut que l'humanité n'y a pas accès. Selon Marx, ce détournement de Dieu serait en quelque sorte la faute de classe originelle, le début de la dramatique série des modes d'aliénation modernes : l'idéalisme philosophique, le pouvoir centralisé de l'État, le droit bourgeois, le capitalisme ... Au terme, l'humanité se trouve privée de tout, de son être même : la capacité de s'humaniser dans et par le travail.

Au début du XXe siècle, dans l'œuvre de Max Weber, l'économie capitaliste est de nouveau mise en relation avec Dieu. En effet, l'accumulation du capital se présente comme l'effet non anticipé, pervers pourrait-on dire, de la recherche dans le protestantisme des justifications tangibles de l'élection et de la prédestination divines ${ }^{5}$. Faut-il le souligner, non seulement L'éthique protestante et l'esprit du capitalisme mais l'ensemble des travaux wébériens de sociologie historique de la religion demeurent à ce jour indépassables ${ }^{6}$. Cependant, c'est l'œuvre de Durkheim sur la religion qui représente bel et bien l'incarnation de Dieu dans la sociologie 7 . On y retrouve, inversés, les termes du drame qui se joue dans l'univers marxien. En effet, selon la théorie durkheimienne, Dieu descend du ciel pour constituer la société en elle-même : il devient le noyau de la conscience collective, siège de la capacité de vivre ensemble. Ainsi, ce que l'humanité adore, ce qui lui demeure sacré, c'est sa propre existence identifiée à la divinité 8 . Certains auteurs, plus proches de nous, ont repris la thèse de Durkheim mais en situant le lieu de la transcendance dans l'existence individuelle. Pour Erwing Goffman,

5 Max WEBER, L'Éthique protestante et l'esprit du capitalisme. Paris, Plon, 1964, (première édition en langue allemande, 1920).

6 Ces travaux sont parus récemment en traduction française, voir Max WEBER, Sociologie des religions. Paris, Gallimard, 1996.

7 Émile DURKHEIM, Les formes élémentaires de la vie religieuse: le système totémique en Australie. Paris, Presses universitaires de France, 1985, (première édition, 1912).

8 Dans le prolongement de l'œuvre durkheimienne, je laisse de côté la «sociologie sacrée» à laquelle œuvrent plusieurs ethnologues, s'inspirant de Marcel Mauss, au cours des années 1930. L'objet de leur démarche serait la "présence active du sacré dans l'existence sociale». Voir l'introduction de Marcel Fournier à Marcel MAUSS, Écrits politiques (Textes réunis et présentés par M. Fournier). Paris, Fayard, 1997, p. 51. 
l'individu demeure en ce sens le seul dieu viable ${ }^{9}$. Selon une perspective semblable, Thomas Luckmann élabore la notion d' « autotranscendance» («self-transcendence»); celle-ci se manifeste par la recherche de l'épanouissement personnel au moyen de la sexualité, des liens familiaux, de l'expression de soi ${ }^{10}$.

Par suite de la différenciation interne de la discipline en secteurs d'étude spécialisés, Dieu quitte le centre de la scène et ses fréquences d'apparition se concentrent dans le voisinage immédiat des religions, des mouvements religieux et des institutions religieuses dont il reste désormais prisonnier. La sociologie de la religion se penche sur des organisations, des pratiques, des discours et des acteurs situés dans un domaine particulier. Celui-ci se définit soit par le territoire qu'il occupe au sein de la société, un peu selon le modèle du "champ» dans la théorie de Bourdieu"1, ou bien encore par la nature et les propriétés des objets qui s'y présentent. Des approches quantitatives aussi bien que des approches qualitatives sont mises en œuvre; les méthodes vont de l'enquête par sondage à l'herméneutique. Peu encline à théoriser pour son propre compte, la sociologie de la religion s'appuie depuis plusieurs années sur les thèses de la sécularisation, reformulées inlassablement selon des terminologies variées par différents théoriciens de la modernité et dernièrement, ceux de la postmodernité : le déclin des religions instituées, la laïcisation de la sphère publique, l'érosion de la culture religieuse, la privatisation de la foi et des pratiques, etc. Banalisées par un usage abusif, quasi-incantatoire, ces thèses expliquent mal la réalité

9 Dans son étude sur les rites d'interaction, Erwing GOFFMAN avance en effet que l'on peut "traduire les notions durkheimiennes quant à la religion primitive en ces concepts que sont la déférence et la tenue, qui nous aident à saisir certains aspects de la vie séculière urbaine. Il s'ensuit qu'en un sens ce monde profane n'est pas aussi irreligieux qu'il y paraît. Bien des dieux ont été mis au rancart, mais l'individu demeure obstinément, déité d'une importance considérable». Voir Les rites d'interaction, Paris, Les Éditions de Minuit, 1974, p. 84.

10 Thomas LUCKMANN, The Invisible Religion. The Problem of Religion in Modern Society. Londres et Toronto, Collier-Macmillan, 1967.

11 L'application de la notion de champ à la sphère religieuse est exposée dans l'article de Pierre BOURDIEU, "Genèse et structure du champ religieux ", Revue française de sociologie XII (1971) 275-334. 
contemporaine. De plus, elles inhibent la réflexion sur le fait reli gieux hors du christianisme et hors des sociétés occidentales. On comprend difficilement la persistance de ce courant de pensée, à moins de faire l'hypothèse qu'il serait l'équivalent, en sociologie, de la mort de Dieu décrétée par certains courants de la philosophie.

Quoi qu'il en soit, la recherche en sociologie de la religion, comme dans plusieurs domaines de la discipline, s'intéresse peu à l'histoire et à la société dans sa globalité. Elle donne plus volontiers priorité à la nouveauté et au changement. Sans exclure la théorisation, sa démarche conduit néanmoins à privilégier des niveaux intermédiaires d'interprétation, très proches des objets d'observation, circonscrivant ainsi la réflexion dans l'espace exigu de la spécialité. Au cours des dernières années, les «nouvelles » formes du religieux les sectes et les fondamentalismes, par exemple - ont fortement retenu l'attention. Les sociologues se sont également penchés sur des croyances et des pratiques inédites, se rattachant notamment à l'ésotérisme et au syncrétisme. En outre, ils se sont intéressés aux nouvelles formes du sacré, lesquelles seraient, paradoxalement, läques et profanes. On le voit, la sociologie de la religion est à la merci de son champ, qui se rétrécit comme une peau de chagrin, et de son objet, apparemment en voie de dénaturation et de disparition. Elle n'a donc plus grand chose à dire sur Dieu sinon qu'il serait victime de la crise du sens, celle-ci se manifestant par la perte, le manque, le vide de sens, associés notamment au déclin de la religion. En contrepartie, la recherche de sens expliquerait la résurgence du fait religieux et de Dieu, sous différents avatars. La base de cette argumentation - la crise du sens présumée - est relativement précaire.

Devant l'impasse apparente du fait religieux, dans la société et dans la sociologie, la tentation de se tourner vers le passé devient forte. En effet, l'histoire religieuse représente un champ de recherche en plein essor et rien n'empêche d'y adapter les méthodes et les problématiques sociologiques ${ }^{12}$. L'histoire nous restitue Dieu

12 Les travaux que j'ai réalisés avec Danielle Juteau sur les communautés religieuses de femmes, au Québec, représentent un exemple de ce type de recherche. Au moyen de l'échantillonnage et de l'analyse statistique, nous avons étudié les caractéristiques socio-démographiques et l'activité professionnelle de 3700 religieuses, issues de vingt-quatre communautés, au cours d'une période de soixante-dix ans. L'interprétation 
dans toute sa splendeur, si on peut dire. Les acteurs sociaux le convoquent et l'invoquent sans inhibition, ils se servent aussi de lui sans scrupule. Pourtant, ce Dieu dissimule souvent le fait socio-historique, il s'interpose comme un écran qu'il faut écarter, le temps d'observer et d'analyser ce qui se passe dans l'histoire, quitte par la suite à inviter Dieu à reprendre sa place qui est partout, alors qu'aujourd'hui, elle n'est nulle part. Il en va ainsi de l'histoire québécoise, de la Nouvelle-France à la Révolution tranquille. Si Dieu n'est pas une idéologie, il s'en distingue parfois fort mal dans l'histoire, au regard de la sociologie. On le voit mobilisé le plus souvent par les groupes conservateurs et, même au nom des pauvres, par ceux qui profitent le mieux de l'ordre (ou du désordre) établi. Les institutions de la régulation et du contrôle social font elles aussi une forte consommation de la divinité.

Sous un autre aspect, Dieu s'intègre souvent dans des doctrines politico-philosophiques, portées par des classes, des partis et des mouvements sociaux. Le catholicisme social en est une et son influence s'est exercée sur la sociologie dans plusieurs sociétés contemporaines, incluant le Québec, jusque dans les années 1960. La sociologie, en effet, est perméable aux courants de pensée qui traversent la société, pensons par exemple au libéralisme, au socialisme, au nationalisme, au féminisme. Elle trouve en eux son inspiration et elle se met parfois à leur service. Les premiers enseignements de sociologie dispensés par la Faculté des sciences sociales de l'Université Laval, et plus tard, celle de l'Université de Montréal, présentent une conception de la société qui se veut compatible avec la pensée de l'Église et sa doctrine sociale. Par exemple, Arthur Saint-Pierre, un laïc, se définit dans les années 1920 et 1930 comme un sociologue catholique.

de ces données se place hors du champ de la sociologie religieuse; elle s'inspire plutôt des problématiques du travail féminin d'une part et des rapports entre les institutions au sein de la société québécoise, d'autre part. Voir, Nicole LAURIN, Danielle JUTEAU et Lorraine DUCHESNE, $\grave{A}$ la recherche d'un monde oublié. Les communautés religieuses de femmes au Québec de 1900 à 1970. Montréal, Le Jour, 1991. Danielle JUTEAU et Nicole LAURIN, Un métier et une vocation. Le travail des religieuses au Québec de 1901 à 1971. Montréal, Les Presses de l'Université de Montréal, 1997. 
Certaines de ses recherches, non dépourvues d'intérêt scientifique, sont explicitement motivées par la défense du syndicalisme catholique, patronal et ouvrier, et par l'illustration du rôle de l'Église au Québec, particulièrement celui des communautés religieuses féminines dans les services sociaux et hospitaliers ${ }^{13}$. Ce sont surtout des clercs, ayant reçu une formation en sociologie, qui les premiers ont vulgarisé les notions fondamentales de la discipline auprès de différents groupes sociaux et professionnels : syndicats, associations patronales, travailleurs sociaux, mouvements de jeunesse, etc. Cependant, au cours des années 1960, le clergé perd son emprise sur la société, sur la politique et sur la vie intellectuelle. Dès lors, un fossé apparem. ment infranchissable se creuse entre l'Église et l'institution universitaire, exception faite des facultés de théologie. La figure de l'intellectuel catholique disparait de la sociologie comme des autres domaines de la pensée scientifique ${ }^{14}$. Pour la plupart des intellectuels, en effet, la pratique scientifique et la foi religieuse sont cloisonnées, lorsqu'elles ne sont pas jugées radicalement incompatibles.

Dieu est désormais absent de la sociologie. Il a quitté d'abord la théorie générale et par la suite, il s'est retiré de la sociologie religieuse. De surcroit, en tant qu'élément de l'idéologie et source de mobilisation intellectuelle et politique, il est tombé en désuétude ${ }^{15}$. Au moment où la théologie chrétienne cherche son inspiration du

13 Arthur SAINT-PIERRE, Le problème social. Quelques éléments de solution. Montréal, Action française, 1925, et L'œuvre des communautés religieuses de charité dans la Province de Québec en 1930. Montréal, Éditions de la Bibliothèque Canadienne, 1930.

14 Sans doute serait-il difficile de concevoir, au Québec, un ouvrage comme celui que Jean Delumeau a publié récemment en France, réunissant les témoignages de vingt-cinq historiens chrétiens qui "à travers leurs divers engagements... évoquent les exigences réciproques de leurs convictions religieuses et de leur discipline». Sous la direction de Jean Delumeau, L'historien et la foi. Paris, Fayard, 1996, p. 4 de couverture.

15 Il faut tout de même mentionner qu'au cours des années 1970 la théologie de la libération a failli réunir les conditions qui auraient pu produire une telle mobilisation d'intellectuels œuvrant dans des perspectives critiques, au sein des sciences sociales. 
côté des sciences et tout particulièrement des sciences humaines, la sociologie n'a donc plus rien à lui offrir. À part le fait social, dépouillé de ses oripeaux, ramené à sa misère et son absurdité. Le fait social dans sa pesanteur ${ }^{16}$. Pesanteur du système et de la régulation; procès sans sujet ni fin de la production et de la reproduction. Pesanteur du langage, circularité du discours, réitération incessante de l'interpellation, l'interdiction et l'injonction. Pesanteur de l'institution et de la hiérarchie, du pouvoir, de l'argent, du signe. Pesanteur de la condition de classe, du sexe, de la race, de la nationalité, qu'ils soient source de dérive ou d'appartenance, d'identité ou de déracinement. La pesanteur n'appartient pas au déterminisme, elle ne s'oppose pas à la liberté. Pour la sociologie, il n'existe en effet ni véritable déterminisme, ni véritable liberté. La vie sociale se crée et se défait sans cesse mais elle n'est nulle part transparente à elle-même dans l'objectivité ou dans la subjectivité des modes de conscience et de discours. Comme toute forme de vie, elle se soutient par l'ordre et le chaos; elle a partie liée avec la mort.

De la présence de Dieu dans cette réalité, la sociologie ne peut rien dire. Elle peut seulement espérer que la théologie pose son regard sur la société, et que ce regard puisse croiser celui de la science, au-delà du fossé épistémologique séparant ces deux domaines de la pensée. Si toutefois la place du discours sur Dieu est vide, en théologie comme en science, il n'y aura plus alors qu'à s'en remettre au silence.

16 "Deux forces règnent sur l'univers: lumière et pesanteur." Simone WEIL, La pesanteur et la grâce. Paris, Plon, 1979, p. 11 (première édition, 1948). 